Contributions to Game Theory and Management, XIII, 8-23

\title{
Dynamic SPICE-Model of Resource Allocation in Marketing Networks ${ }^{\star}$
}

\author{
Movlatkhan T. Agieva $^{1}$ and Olga I. Gorbaneva ${ }^{2}$ \\ 1 Ingush State University, \\ Magistralnaya St. 39, Nazran, 386132, Russia \\ E-mail: agieva25@mail.ru \\ 2 South Federal University, \\ J.I. Vorovich Institute of Mathematics, Mechanics and Computer Sciences, \\ Milchakov St. 8a, Rostov-on-Don, 344090, Russia \\ E-mail: gorbaneva@mail.ru
}

\begin{abstract}
We consider a dynamic Stackelberg game theoretic model of the coordination of social and private interests (SPICE-model) of resource allocation in marketing networks. The dynamics of controlled system describes an interaction of the members of a target audience (basic agents) that leads to a change of their opinions (cost of buying the goods and services of firms competing on a market). An interaction of the firms (influence agents) is formalized as their differential game in strategic form. The payoff functional of each firm includes two terms: the summary opinion of the basic agents with consideration of their marketing costs (a common interest of all firms), and the income from investments in a private activity. The latter income is described by a linear function. The firms exert their influence not to all basic agents but only to the members of strong subgroups of the influence digraph (opinion leaders). The opinion leaders determine the stable final opinions of all members of the target audience. A coordinating principal determines the firms' marketing budgets and maximizes the summary opinion of the basic agents with consideration of the allocated resources. The Nash equilibrium in the game of influence agents and the Stackelberg equilibrium in a general hierarchical game of the principal with them are found. It is proved that the value of opinion of a basic agent is the same for all influence agents and the principal. It is also proved that the influence agents assign less resources for the marketing efforts than the principal would like.
\end{abstract}

Keywords: differential Stackelberg games, marketing, opinion control on networks, resource allocation.

\section{Introduction}

Models of influence and opinion control on networks are widespread in the literature in the last several decades (Chkhartishvili, Gubanov and Novikov, 2019; Jackson, 2008). The approach most close to the authors' one is presented in the papers (Sedakov and Zhen, 2019; Zhen, 2019). In those models a network is described by a weighted directed graph in which the vertices represent the members of the network (basic agents), and the weights of the arcs reflect an intensity of their mutual influence. The basic agents have their opinions about an issue which can change in time due to the network interaction. External influence agents can impact the basic agents in their own interests. We suppose the following. First, it is rational to exert control impact not to all basic agents but only to the members

\footnotetext{
${ }^{\star}$ The work was supported by the Russian Science Foundation, project № 17-19-01038.
} 
of strong subgroups of the influence digraph (opinion leaders). It is known that the opinion leaders determine the stable final opinions of all basic agents. Second, we consider marketing networks in which the opinions of basic agents are their costs of buying of the goods and services providing by firms competing on a market (influence agents), and control variables of the influence agents are their marketing efforts directed to the opinion leaders (Agieva, Korolev and Ougolnitsky, 2019).

Models of coordination of the social and private interests, including their dynamic formulation, are studied in the public economics (Long, 2010). In those models it is supposed that each agent divides his efforts between a private activity and the production of a good which is common for all agents. In turn, the agent's payoff is a sum of the income from his private activity and his share in the utility from the production of the common good. The author's formulation of the models of coordination of social and private resources (SPICE-models) is given in (Gorbaneva, 2019), and some examples of the application of SPICE-models in different problem domains are described in (Anopchenko and others, 2019a; Anopchenko and others, 2019b; Sukhinov, Ougolnitsky and Usov, 2020; Ugol'nitskii and Usov, 2020).

An important aspect of the control in complex systems consists in the evaluation of a degree of coordination between the active agents. The most known formulation is the problem of inefficiency of equilibria when the global maximal value of the function of social welfare is compared with its value in the worst of Nash equilibria in a game of the agents (Nisan, Roughgarden, Tardos and Vazirani, 2007).

This paper is based on the mentioned streams of research and makes the following contribution. We consider a hierarchical dynamic SPICE-model of resource allocation in a marketing network. The dynamics of opinions of the basic agents (members of the target audience) is determined by their interaction and marketing efforts of several competing firms (influence agents) that form the lower control level. The payoff functional of each firm includes two terms: the summary opinion of all basic agents with consideration of the marketing cost (a common interest of all influence agents), and the income from investments in a private activity. The latter income is described by a linear function. It is assumed that in the stage of analysis of the network the members of its strong subgroups (opinion leaders) are already found, and the firms exert marketing influence only on them. On the upper control level a coordinating principal is situated. The principal allocates marketing budgets between the firms (influence agents) for the maximization of the summary opinion of the basic agents. The unique Nash equilibrium in the game of influence agents in strategic form and the unique Stackelberg equilibrium in the game of the principal with them are found. The evaluation of the degree of coordination between the principal and the influence agents is made by means of a special system compatibility index (Sukhinov, Ougolnitsky and Usov, 2020).

In Section 2 we describe the SPICE-model of resource allocation in marketing networks, precise its specifics, and characterize the used methods. In Section 3 the Nash equilibrium in the differential game of influence agents in strategic form is built. In Section 4 the solution of the differential Stackelberg game between the principal and the influence agents is built. In section 5 the system compatibility index is calculated. Section 6 concludes and contains the directions of future research. 


\section{Dynamical Hierarchical SPICE-Model}

The hierarchical dynamic SPICE-model of resource allocation in a marketing network has the form:

$$
\begin{aligned}
& J_{0}\left(\left\{r_{i}\right\}_{i=1}^{m},\left\{\left\{u_{j}^{i}\right\}_{j=1}^{n}\right\}_{i=1}^{m},\left\{x_{j}\right\}_{j=1}^{n}\right)= \\
& =\int_{0}^{T} e^{-\rho t}\left[\sum_{j=1}^{n} x_{j}(t)-\sum_{i=1}^{m} r_{i}(t)\right] d t \rightarrow \max \\
& r_{i}(t) \geq 0, \sum_{k=1}^{m} r_{k}(t) \leq R \\
& J_{i}\left(r_{i},\left\{u_{j}^{i}\right\}_{j=1}^{n},\left\{x_{j}\right\}_{j=1}^{n}\right)= \\
& =\int_{0}^{T} e^{-\rho t}\left(p_{i}\left(r_{i}(t)-\sum_{j=1}^{n} u_{j}^{i}(t)\right)+\sum_{j=1}^{n}\left[x_{j}(t)-s_{j}^{i} u_{j}^{i}(t)\right]\right) d t \rightarrow \max \\
& 0 \leq \sum_{j=1}^{n} u_{j}^{i}(t) \leq r_{i}(t), i=1, \ldots, m ; t \in[0, T] \\
& \dot{x}_{j}=\sum_{i=1}^{m} b_{j}^{i} \sqrt{u_{j}^{i}(t)}+\sum_{l=1}^{n} a_{l j} x_{l}(t), x_{j}(0)=x_{j 0}, j=1, \ldots, n, \\
& s_{j}^{i}= \begin{cases}1, & b_{j}^{i}>0 \\
0, & b_{j}^{i}=0 .\end{cases}
\end{aligned}
$$

Thus, (1)-(6) is a differential Stackelberg game of the principal with several influence agents (firms). Here $n$ is a number of basic agents (a number of target audience), $m$ - a number of influence agents (competing firms), $R$ - a total marketing budget of the leader, $T$ - a length of the game, $J_{0}, J_{i}$ - the payoff functionals of the leader and the influence agents respectively, $r_{i}(t)$ - a marketing budget allocated to the $i$-th influence agent by the leader in the moment of time $t, x_{j}(t)-$ an opinion of the $j$-th basic agent in the moment $t$ (cost of buying of goods and services), $u_{j}^{i}(t)-$ expenditures of the $i$-th influence agent for the marketing impact (advertizing and so on) to the $j$-th basic agent in the moment $t, a_{i j}$ - a coefficient of influence of the $i$-th basic agent to the $j$-th basic agent, $b_{j}^{i}$ - a coefficient of influence of the $i$-th influence agent to the $j$-th basic agent, $\delta$ denotes a discount factor, i.e. $\delta=e^{-\rho}$. As different firms can exert influence to different members of the strong subgroups, we simply assume that if the $i$-th firm (influence agent) does not impact to the $j$-th basic agent then $b_{j}^{i}=0, s_{j}^{i}$ characterizes the connection of the $i$-th agent of influence and the $j$-th basic agent.

The Principal has at any instant of time $t$ a value of resources to be allocated to the influence agents. Each of the agents receives a value of resources $r_{i}(t)$ that is a continuous function $r_{i}(t):[0, T] \rightarrow[0, R]$. The budget constraint means that $\sum_{i=1}^{m} r_{i}(t) \leq R$. After receiving the resources $r_{i}(t)$ from the Principal the influence agents use them in private and common interests. The common interests are modeled by the function $u_{j}^{i}(t)$ which describes the share of the $i$-th influence agent's resources assigned for the marketing impact on the $j$-th basic agent at the moment $t$. The continuous functions $u_{j}^{i}(t):[0, T] \rightarrow\left[0, r_{i}(t)\right]$ are the strategies of the $i$-th influence 
agent which satisfy the budget constraint $\sum_{j=1}^{n} u_{j}^{i}(t)<r_{i}(t)$. The marketing impact includes advertizing, special actions, discounts, gifts, and so on.

A common (social) interest of the influence agents is modeled as maximization of the summary opinion of the basic agents, and their private interests are represented by the income from an activity which is not concerned with marketing efforts (for example, the private investments). We used the linear functions of the private activity, i.e. $p_{i}(x)=p_{i} \cdot x$, where $p_{i}>0$ is a constant value. In turn, the principal maximizes the summary opinion of all basic agents minus the cost of resources allocated to the firms.

The unique Nash equilibrium in the game of influence agents in strategic form and the unique Stackelberg equilibrium in the game of the principal with them are found analytically by the Hamilton-Jacobi-Bellman equations.

\section{Building the Nash Equilibrium in the Game of Influence Agents}

Let us investigate the problem of the $i$-th firm (3)-(6). The Hamilton-JacobiBellman equation is

$$
\begin{array}{r}
\rho V_{i}-\frac{\partial V_{i}}{\partial t}=\max _{u_{j}^{i}, 1 \leq j \leq n}\left\{\sum_{j=1}^{n}\left[x_{j}(t)-s_{j}^{i} u_{j}^{i}(t)\right]+p_{i}\left(r_{i}(t)-\sum_{j=1}^{n} u_{j}^{i}(t)\right)+\right. \\
\left.+\sum_{q=1}^{n} \frac{\partial V_{i}}{\partial x_{q}}\left[\sum_{k=1}^{m} b_{q}^{k} \sqrt{u_{q}^{k}(t)}+\sum_{l=1}^{n} a_{l q} x_{l}\right]\right\}
\end{array}
$$

with condition $0 \leq \sum_{j=1}^{n} u_{j}^{i}(t) \leq r_{i}(t)$. Maximization by $u_{j}^{i}, j=1,2, \ldots, n, b_{j}^{i} \neq 0$ gives

$$
u_{j}^{i}(t)=\frac{R_{i}(t)\left(b_{j}^{i} \frac{\partial V_{i}}{\partial x_{j}}\right)^{2}}{\sum_{j: b_{j}^{i} \neq 0}\left(b_{j}^{i} \frac{\partial V_{i}}{\partial x_{j}}\right)^{2}},
$$

where $\sum_{j: b_{j}^{i} \neq 0} u_{j}^{i}(t)=R_{i}(t) \leq r_{i}(t)$.

Notice that we can substitute the expression $u_{j}^{i}(t)=\frac{R_{i}(t)\left(b_{j}^{i} \frac{\partial V_{i}}{\partial x_{j}}\right)^{2}}{\sum_{j: b_{j}^{i} \neq 0}\left(b_{j}^{i} \frac{\partial V_{i}}{\partial x_{j}}\right)^{2}}$ by $u_{j}^{i}(t)=$ $=\frac{R_{i}(t)\left(b_{j}^{i} \frac{\partial V_{i}}{\partial x_{j}}\right)^{2}}{\sum_{j=1}^{n}\left(b_{j}^{i} \frac{\partial V_{i}}{\partial x_{j}}\right)^{2}}$, and the expression $R_{i}(t)=\sum_{j: b_{j}^{i} \neq 0} u_{j}^{i}(t)$ by $R_{i}(t)=\sum_{j=1}^{n} u_{j}^{i}(t)$, as if $b_{j}^{i}=0$ then the summand $\left(b_{j}^{i} \frac{\partial V_{i}}{\partial x_{j}}\right)^{2}$ is equal to zero and can be inserted in the common sum. Therefore, $\sum_{j=1}^{n}\left(b_{j}^{i} \frac{\partial V_{i}}{\partial x_{j}}\right)^{2}=\sum_{j: b_{j}^{i} \neq 0}\left(b_{j}^{i} \frac{\partial V_{i}}{\partial x_{j}}\right)^{2}$. Similarly, $\sum_{j=1}^{n} u_{j}^{i}(t)=\sum_{j: b_{j}^{i} \neq 0} u_{j}^{i}(t)$, as if $b_{j}^{i}=0$ then $u_{j}^{i}(t)=0$. We consider the case when the product of all $b_{j}^{i}$ is not equal to zero, otherwise the problem has no practical sense (there is no influence).

Let us use the linear Bellman functions

$$
V_{i}(x, t)=\sum_{j=1}^{n} \alpha_{j}^{i}(t) x_{j}+\beta^{i}(t),
$$


then we can write the equation (7) with consideration of (8) as

$$
\begin{aligned}
& \rho \sum_{j=1}^{n} \alpha_{j}^{i}(t) x_{j}+\rho \beta^{i}(t)-\sum_{j=1}^{n} \alpha_{j}^{i}(t) x_{j}-\rho \beta^{\prime i}(t)= \\
& =\sum_{j=1}^{n} x_{j}(t)-R_{i}(t)+p_{i} \cdot\left(r_{i}(t)-\sum_{j=1}^{n} u_{j}^{i}(t)\right)+\sum_{l=1}^{n} \sum_{j=1}^{n} \alpha_{j}^{i}(t) a_{l j} x_{l}+ \\
& +\sum_{k=1}^{m} \sum_{j=1}^{n} \alpha_{j}^{i}(t) b_{j}^{k} \frac{\sqrt{\left(R_{k}(t)\right.}\left|\alpha_{j}^{k}(t) b_{j}^{k}\right|}{\sqrt{\sum_{j=1}^{n}\left(\alpha_{j}^{k}(t) b_{j}^{k}\right)^{2}}} .
\end{aligned}
$$

Equating in the left and right hand sides of the equation (9) the coefficients at the first power of $x$, we receive the following differential equations for the coefficients $\alpha$ :

$$
\rho \alpha_{j}^{i}(t)-\alpha_{j}^{\prime i}(t)=1+\sum_{l=1}^{n} \alpha_{l}^{i}(t) a_{j l}, j=1,2, \ldots, n .
$$

Rewrite the system of equations (10) in the matrix form

$$
\alpha^{\prime j}=(\rho I-A) \alpha^{j}-\epsilon,
$$

where $A=\left\{a_{i j}\right\}_{i=1,2, \ldots, n ; j=1,2, \ldots, n}$ is the influence matrix, $\alpha^{i}$ - the column vector of the coefficients $\alpha_{j}^{i}, j=1,2, \ldots, n, I$ - the unit matrix, $\epsilon-n$-dimensional column vector of units. System (11) is the same for all influence agents, therefore $\alpha_{j}^{1}(t)=$ $=\alpha_{j}^{2}(t)=\ldots=\alpha_{j}^{m}(t)=\alpha_{j}(t)$ for any basic agent $j=1,2, \ldots, n$, and from this point we will omit the superscript $i$ of the coefficients $\alpha_{j}$.

Solving the system of differential equations (10), we receive:

$$
\begin{array}{r}
\bar{\alpha}=(\rho I-A)^{-1} \epsilon, \\
\alpha=e^{(\rho I-A) t} C+(\rho I-A)^{-1} \epsilon .
\end{array}
$$

The column vector of the constants of integration is found from the boundary conditions:

$$
\alpha(T)=0
$$

therefore

$\alpha=-e^{(\rho I-A)(t-T)}(\rho I-A)^{-1} \epsilon+(\rho I-A)^{-1} \epsilon=\left(e^{(\rho I-A)(T-t)}-I\right)(A-\rho I)^{-1} \epsilon$.

In particular, for $t=0$ we have

$$
\alpha(0)=\left(e^{(\rho I-A) T}-I\right)(A-\rho I)^{-1} \epsilon .
$$

Considering that $\alpha_{j}^{i}(t)=\alpha_{j}^{k}(t)$ for any $k=1,2, \ldots, n$, we rewrite $(9)$ in the form:

$$
\begin{array}{r}
\rho \sum_{j=1}^{n} \alpha_{j}^{i}(t) x_{j}+\rho \beta^{i}(t)-\sum_{j=1}^{n} \alpha_{j}^{\prime i}(t) x_{j}-\beta^{\prime i}(t)=\sum_{j=1}^{n} x_{j}(t)-R_{i}(t)+ \\
+p_{i} \cdot\left(r_{i}(t)-R_{i}(t)\right)+\sum_{l=1}^{n} \sum_{j=1}^{n} \alpha_{j}^{i}(t) a_{l j} x_{l}+\sum_{k=1}^{m} \sqrt{R_{k}(t)} \sqrt{\sum_{j=1}^{n}\left(\alpha_{j}^{k}(t) b_{j}^{k}\right)^{2}}
\end{array}
$$


Choosing the maximal value of the right hand side of (7) in dependence of the sum $R_{i}(t)$, we have

$$
-R_{i}(t)+p_{i} \cdot\left(r_{i}(t)-R_{i}(t)\right)+\sqrt{R_{i}(t)} \sqrt{\sum_{j=1}^{n}\left(\alpha_{j}^{i}(t) b_{j}^{i}\right)^{2}} \rightarrow \max ,
$$

and

$$
\left(R_{i}(t)\right)_{\max }=\frac{\sum_{j=1}^{n}\left(b_{j}^{i} \alpha_{j}^{i}(t)\right)^{2}}{4\left(1+p_{i}\right)^{2}} .
$$

Thus, the value $R_{i}(t)$ in (13) with consideration of (4) is equal to

$$
R_{i}(t)=\min \left\{\frac{\sum_{j=1}^{n}\left(b_{j}^{i} \alpha_{j}^{i}(t)\right)^{2}}{4\left(1+p_{i}\right)^{2}} ; r_{i}(t)\right\} .
$$

Let us call the amount $\alpha_{j}^{i}(t)$ the opinion value of the $j$-th basic agent for the $i$-th influence agent. In fact, in the Bellman function of the $i$-th influence agent $V_{i}(x, t)=\sum_{j=1}^{n} \alpha_{j}^{i}(t) x_{j}+\beta^{i}(t)$, the factor $\alpha_{j}^{i}(t)$ is a weight coefficient of the opinion of the respective basic agent $x_{j}$. Thus, from the condition $\alpha_{j}^{i}(t)=\alpha_{j}^{k}(t)$ we receive

Proposition 1. The opinion value of each basic agent is the same for all influence agents.

Equating the constant terms in the left and right hand sides of (9), we receive the differential equation for $\beta^{i}$ :

$$
\beta^{\prime i}(t)-\rho \beta^{i}(t)=-\sum_{k=1}^{m} \sqrt{R_{k}(t)} \sqrt{\sum_{j=1}^{n}\left(\alpha_{j}(t) b_{j}^{k}\right)^{2}}+p_{i} R_{i}(t)+R_{i}(t) .
$$

The equation (14) is solved by the method of variation of parameters:

$$
\beta^{i}(t)=\int_{t}^{T} e^{-\rho(\tau-t)}\left\{\sum_{k=1}^{m} \sqrt{R_{k}(\tau) \sum_{j=1}^{n}\left(\alpha_{j}(\tau) b_{j}^{k}\right)^{2}}-\left(p_{i}+1\right) R_{i}(\tau)\right\} d \tau .
$$

For $t=0$ we have

$$
\beta^{i}(0)=\int_{0}^{T} e^{-\rho \tau}\left\{\sum_{k=1}^{m} \sqrt{R_{k}(\tau) \sum_{j=1}^{n}\left(\alpha_{j}(\tau) b_{j}^{k}\right)^{2}}-\left(p_{i}+1\right) R_{i}(\tau)\right\} d \tau,
$$

where for any $k=1,2, \ldots, n$ (in particular, for the given $i$ ) we have

$$
R_{k}(t)= \begin{cases}\frac{\sum_{j=1}^{n}\left(b_{j}^{k} \alpha_{j}(t)\right)^{2}}{4\left(1+p_{k}\right)^{2}} ; & \sum_{j=1}^{n}\left(b_{j}^{k} \alpha_{j}(t)\right)^{2} \leq 4\left(1+p_{k}\right)^{2} r_{k}(t), \\ r_{k}(t), & \sum_{j=1}^{n}\left(b_{j}^{k} \alpha_{j}(t)\right)^{2} \leq 4\left(1+p_{k}\right)^{2} r_{k}(t) .\end{cases}
$$

Thus, the maximal payoff of the influence agent is

$$
\begin{array}{r}
\max _{u_{j}^{i}, 1 \leq j \leq n} J_{i}=V_{i}(x(0), 0)=\sum_{j=1}^{n} \alpha_{j}(0) x_{j}(0)+ \\
+\int_{0}^{T} e^{-\rho \tau}\left\{\sum_{k=1}^{m} \sqrt{R_{k}(\tau) \sum_{j=1}^{n}\left(\alpha_{j}(\tau) b_{j}^{k}\right)^{2}}-\left(p_{i}+1\right) R_{i}(\tau)\right\} d \tau,
\end{array}
$$


where the components $\alpha_{j}(0), j=1,2, \ldots, n$ of the vector $\alpha(0)$ are determined by the expression (12), and $R_{k}(\tau), k=1,2, \ldots, m$ are determined by the expression (15). The control values are determined by the expression (8):

$$
u_{j}^{i}(t)=\frac{R_{i}(t)\left(b_{j}^{i} \alpha_{j}(t)\right)^{2}}{\sum_{j=1}^{n}\left(b_{j}^{i} \alpha_{j}(t)\right)^{2}},
$$

where

$$
\begin{gathered}
\alpha(t)=\left(e^{(A-\rho I)(T-t)}-I\right)(A-\rho I)^{-1} \epsilon, \\
R_{i}(t)=\min \left\{\frac{\sum_{j=1}^{n}\left(b_{j}^{i} \alpha_{j}^{i}(t)\right)^{2}}{4\left(1+p_{i}\right)^{2}} ; r_{i}(t)\right\} .
\end{gathered}
$$

\section{The Stackelberg Game of the Principal with the Influence Agents}

Let us move to the principal's problem (1)-(2),(5)-(6). The Hamilton-JacobiBellman equation has the form

$$
\begin{array}{r}
\rho V_{0}-\frac{\partial V_{0}}{\partial t}=\max _{r_{i}(t), 1 \leq i \leq m}\left\{\sum_{j=1}^{n} x_{j}(t)-\sum_{i=1}^{m} r_{i}(t)+\right. \\
\left.+\sum_{j=1}^{n} \frac{\partial V_{0}}{\partial x_{j}}\left[\sum_{k=1}^{m} b_{j}^{k} \sqrt{u_{j}^{k}(t)}+\sum_{l=1}^{n} a_{l j} x_{l}\right]\right\}
\end{array}
$$

with conditions $0 \leq \sum_{i=1}^{m} r_{i}(t) \leq R, r_{i}(t) \geq 0, i=1,2, \ldots, m$, where

$$
u_{j}^{i}(t)=\min \left\{\frac{\left(b_{j}^{i} \alpha_{j}(t)\right)^{2}}{4\left(1+p_{i}\right)^{2}} ; \frac{r_{i}(t)\left(b_{j}^{i} \alpha_{j}(t)\right)^{2}}{\sum_{j=1}^{n}\left(b_{j}^{i} \alpha_{j}(t)\right)^{2}}\right\} .
$$

The equation (16) takes the form:

$$
\begin{aligned}
\rho V_{0} & -\frac{\partial V_{0}}{\partial t}=\max _{r_{i}(t), 1 \leq i \leq m}\left\{\sum_{j=1}^{n} x_{j}(t)-\sum_{i=1}^{m} r_{i}(t)+\sum_{j=1}^{n} \frac{\partial V_{0}}{\partial x_{j}}\left[\sum_{l=1}^{n} a_{l j} x_{l}+\right.\right. \\
& \left.\left.+\sum_{i=1}^{m} b_{j}^{i} \frac{\left|b_{j}^{i} \alpha_{j}(t)\right|}{\sqrt{\sum_{j=1}^{n}\left(b_{j}^{i} \alpha_{j}(t)\right)^{2}}} \min \left\{\sqrt{\frac{1}{4} \sum_{j=1}^{n}\left(b_{j}^{i} \alpha_{j}(t)\right)^{2}} ; \sqrt{r_{i}(t)}\right\}\right]\right\} .
\end{aligned}
$$

Let us take the linear Bellman function

$$
V_{0}(x, t)=\sum_{j=1}^{n} \alpha_{j}^{0}(t) x_{j}+\beta^{0}(t) .
$$

Let us show that $\alpha_{j}^{0}(t)=\alpha_{j}(t), j=1,2, \ldots, n, t \in[0, T]$. Equating in (17) the coefficients at the first power of $x_{j}$ in the left and right hand sides, we receive the differential equation for $\alpha_{j}^{0}(t)$ :

$$
\alpha_{j}^{\prime 0}(t)-\rho \alpha_{j}^{0}(t)+\sum_{l=1}^{n} \alpha_{l}^{0}(t) a_{j l}=-1,
$$


which coincides with (10) for the same boundary conditions

$$
\alpha(T)=0,
$$

therefore

$$
\alpha_{j}^{0}(t)=\alpha_{j}^{1}(t)=\ldots=\alpha_{j}^{m}(t)=\alpha_{j}(t), j=1,2, \ldots, n, t \in[0 ; T] .
$$

Let us call $\alpha_{j}^{0}(t)$ the opinion value of the $j$-th basic agent for the principal. In the principal's Bellman function $V_{0}(x, t)=\sum_{j=1}^{n} \alpha_{j}^{0}(t) x_{j}+\beta^{0}(t)$ the factor $\alpha_{j}^{0}(t)$ is the weight coefficient of the opinion of the respective basic agent. Thus, from the condition (18) we receive

Proposition 2. The opinion value of each basic agent is the same for all influence agents and the principal.

With consideration of (18) we can rewrite (17) as

$$
\begin{array}{r}
\rho\left(\beta^{0}+\sum_{j=1}^{n} \alpha_{j} x_{j}\right)-\left(\beta^{\prime 0}+\sum_{j=1}^{n} \alpha_{j}^{\prime} x_{j}\right)= \\
=\max _{r_{i}(t), 1 \leq i \leq m}\left\{\sum_{j=1}^{n} x_{j}(t)-\sum_{i=1}^{m} r_{i}(t)+\sum_{i=1}^{n} \sum_{j=1}^{n} \alpha_{j}(t) a_{i j} x_{i}+\right. \\
\left.+\sum_{j=1}^{n} \sum_{i=1}^{m} \frac{\left(\alpha_{j}(t) b_{j}^{i}\right)^{2}}{\sqrt{\sum_{j=1}^{n}\left(\alpha_{j}(t) b_{j}^{i}\right)^{2}}} \min \left\{\frac{1}{2\left(1+p_{i}\right)} \sqrt{\sum_{j=1}^{n}\left(b_{j}^{i} \alpha_{j}(t)\right)^{2}} ; \sqrt{r_{i}(t)}\right\}\right\} .
\end{array}
$$

If the minimum is attained in the first expression, the function decreases on $r_{i}(t)$, and the optimal value of $r_{i}(t)$ is minimal. It is minimal if the expressions under the sign of minimum are equal. Thus, the minimum is attained in the second expression or it is absent.

Notice that the non-conditional maximization of the expression (19) gives the amount of resources

$$
r_{i}(t)=\frac{1}{4} \sum_{j=1}^{n}\left(b_{j}^{i} \alpha_{j}(t)\right)^{2} .
$$

In this case

$$
u_{j}^{i}(t)=\min \left\{\frac{1}{4\left(1+p_{i}\right)^{2}}\left(b_{j}^{i} \alpha_{j}(t)\right)^{2} ; \frac{\left(b_{j}^{i} \alpha_{j}(t)\right)^{2}}{4}\right\}=\frac{1}{4\left(1+p_{i}\right)^{2}}\left(b_{j}^{i} \alpha_{j}(t)\right)^{2} .
$$

It means that it is not advantageous for the principal to allocate the amount of resources

$$
r_{i}(t)=\frac{1}{4} \sum_{j=1}^{n}\left(b_{j}^{i} \alpha_{j}(t)\right)^{2} .
$$

Thus, the principal allocates to an influence agent as many resources as the latter is ready to assign for the common purposes, i.e.

$$
r_{i}(t)=\frac{1}{4\left(1+p_{i}\right)^{2}} \sum_{j=1}^{n}\left(b_{j}^{i} \alpha_{j}(t)\right)^{2} .
$$


and we receive the following

Proposition 3. The influence agents assign less resources for the marketing efforts than the principal would like.

Thus if

$$
\sum_{i=1}^{m} \frac{\sum_{j=1}^{n}\left(b_{j}^{i} \alpha_{j}(t)\right)^{2}}{\left(1+p_{i}\right)^{2}} \leq 4 R
$$

then the principal allocates to each agent the amount of resources equal to (20). How the principal should allocate the resources if $\sum_{i=1}^{m} \frac{\sum_{j=1}^{n}\left(b_{j}^{i} \alpha_{j}(t)\right)^{2}}{\left(1+p_{i}\right)^{2}}>4 R$ ? The answer is given by the conditional maximization of the expression (19). Maximizing the right hand side of $(19)$ by $r_{i}(t), i=1,2, \ldots, m$ with condition $\sum_{i=1}^{n} r_{i}(t) \leq R$, we receive

$$
r_{i}(t)=R \frac{\sum_{j=1}^{n}\left(\alpha_{j}(t) b_{j}^{i}\right)^{2}}{\sum_{i=1}^{m} \sum_{j=1}^{n}\left(\alpha_{j}(t) b_{j}^{i}\right)^{2}} .
$$

Thus, in the instants $t \in[0, T]$ when

$$
\sum_{i=1}^{m} \frac{\sum_{j=1}^{n}\left(b_{j}^{i} \alpha_{j}(t)\right)^{2}}{\left(1+p_{i}\right)^{2}} \leq 4 R
$$

the optimal control of the principal is

$$
r_{i}(t)=\frac{1}{4\left(1+p_{i}\right)^{2}} \sum_{j=1}^{n}\left(b_{j}^{i} \alpha_{j}(t)\right)^{2}, i=1,2, \ldots, m,
$$

and in the instants $t \in[0, T]$ when

$$
\sum_{i=1}^{m} \frac{\sum_{j=1}^{n}\left(b_{j}^{i} \alpha_{j}(t)\right)^{2}}{\left(1+p_{i}\right)^{2}}>4 R
$$

the optimal control of the principal is

$$
r_{i}(t)=\frac{\sum_{j=1}^{n}\left(\alpha_{j}(t) b_{j}^{i}\right)^{2}}{\sum_{i=1}^{m} \sum_{j=1}^{n}\left(\alpha_{j}(t) b_{j}^{i}\right)^{2}} R, i=1,2, \ldots, m .
$$

Combining both expressions for $r_{i}(t)$ in the same formula, we receive the final optimal control of the principal:

$$
\begin{aligned}
& r_{i}(t)=\frac{\sum_{j=1}^{n}\left(\alpha_{j}(t) b_{j}^{i}\right)^{2}}{\sum_{k=1}^{m} \sum_{j=1}^{n}\left(\alpha_{j}(t) b_{j}^{k}\right)^{2}} \min \left\{\frac{1}{4\left(1+p_{i}\right)^{2}} \sum_{k=1}^{m} \sum_{j=1}^{n}\left(\alpha_{j}(t) b_{j}^{k}\right)^{2} ; R\right\}, \\
& i=1,2, \ldots, m, t \in[0, T],
\end{aligned}
$$

where

$$
\alpha(t)=\left(e^{(A-\rho I)(T-t)}-I\right)(A-\rho I)^{-1} \epsilon
$$


Given (21) we can simplify (19) because

$$
\sum_{i=1}^{m} r_{i}(t)=\min \left\{\frac{1}{4} \sum_{i=1}^{m} \frac{\sum_{j=1}^{n}\left(b_{j}^{i} \alpha_{j}(t)\right)^{2}}{\left(1+p_{i}\right)^{2}}, R\right\}
$$

and the last term in (19) is

$$
\begin{array}{r}
\sum_{i=1}^{m} \sum_{j=1}^{n} \frac{\left(\alpha_{j}(t) b_{j}^{i}\right)^{2}}{\sqrt{\sum_{j=1}^{n}\left(\alpha_{j}(t) b_{j}^{i}\right)^{2}}} \min \left\{\frac{1}{2\left(1+p_{i}\right)} \sqrt{\sum_{j=1}^{n}\left(\alpha_{j}(t) b_{j}^{i}\right)^{2}} ; \sqrt{r_{i}(t)}\right\}= \\
=\left\{\begin{array}{cl}
\frac{1}{2} \sum_{i=1}^{m} \frac{\sum_{j=1}^{n}\left(b_{j}^{i} \alpha_{j}(t)\right)^{2}}{1+p_{i}}, & \sum_{i=1}^{m} \frac{\sum_{j=1}^{n}\left(b_{j}^{i} \alpha_{j}(t)\right)^{2}}{\left(1+p_{i}\right)^{2}} \leq 4 R, \\
\sqrt{R \sum_{i=1}^{m} \sum_{j=1}^{n}\left(b_{j}^{i} \alpha_{j}(t)\right)^{2}}, \sum_{i=1}^{m} \frac{\sum_{j=1}^{n}\left(b_{j}^{i} \alpha_{j}(t)\right)^{2}}{\left(1+p_{i}\right)^{2}}>4 R .
\end{array}\right.
\end{array}
$$

Equating the constant terms in the left and right hand sides of (19), we receive the differential equation for $\beta^{0}(t)$ :

$$
\beta^{\prime 0}(t)-\rho \beta^{0}(t)=f(t),
$$

where

$$
f(t)=\left\{\begin{array}{c}
\frac{1}{4} \sum_{i=1}^{m} \sum_{j=1}^{n}\left(b_{j}^{i} \alpha_{j}(t)\right)^{2} \frac{2 p_{i}^{2}+p_{i}+1}{\left(1+p_{i}\right)^{2}}, \sum_{i=1}^{m} \frac{\sum_{j=1}^{n}\left(b_{j}^{i} \alpha_{j}(t)\right)^{2}}{\left(1+p_{i}\right)^{2}} \leq 4 R, \\
\sqrt{R \sum_{i=1}^{m} \sum_{j=1}^{n}\left(b_{j}^{i} \alpha_{j}(t)\right)^{2}}-R, \quad \sum_{i=1}^{m} \frac{\sum_{j=1}^{n}\left(b_{j}^{i} \alpha_{j}(t)\right)^{2}}{\left(1+p_{i}\right)^{2}}>4 R .
\end{array}\right.
$$

Solving the equation (22) by the method of variation of parameters, we receive:

$$
\beta^{0}(t)=\int_{t}^{T} e^{-\rho(\tau-t)} f(\tau) d \tau .
$$

When $t=0$ we have

$$
\beta^{0}(0)=\int_{0}^{T} e^{-\rho \tau} f(\tau) d \tau .
$$

Thus, the maximal guaranteed payoff of the principal is

$$
\max _{r_{i}, 1 \leq i \leq m} J_{0}=V_{0}(x(0), 0)=\sum_{j=1}^{n} \alpha_{j}(0) x_{j}(0)+\int_{0}^{T} e^{-\rho \tau} f(\tau) d \tau,
$$

where the components $\alpha_{j}(0), j=1,2, \ldots, n$ of the vector $\alpha(0)$ are determined by the expression (12), and $f(\tau)$ is determined by the expression (23).

\section{System Compatibility Index}

Now let us calculate the system compatibility index:

$$
S C I=\frac{\max _{r_{i}(t)} \min _{u_{j}^{i}(t) \in N E\left(r_{i}(t)\right)} J_{0}\left(\left\{r_{i}\right\}_{i=1}^{m},\left\{\left\{u_{j}^{i}\right\}_{j=1}^{n}\right\}_{i=1}^{m},\left\{x_{j}\right\}_{j=1}^{n}\right)}{\max _{r_{i}(t)} \max _{u_{j}^{i}(t)} J_{0}\left(\left\{r_{i}\right\}_{i=1}^{m},\left\{\left\{u_{j}^{i}\right\}_{j=1}^{n}\right\}_{i=1}^{m},\left\{x_{j}\right\}_{j=1}^{n}\right)},
$$

where the set $N E\left(r_{i}(t)\right)$ is the Nash equillibrium of the agents of influence in responce to the resource amount $r_{i}(t)$. 
This index shows the measure of compatability of system. The numerator of (25) is the payoff of the principal in the case if the firms choose the most unfavorable for principal equilibrium strategies. The denumerator of (25) is the payoff of the principal in the case if the firms choose the most favorable for principal strategies. It is evident that $0 \leq S C I \leq 1$. A similar formula is used in the paper (Agieva, Korolev and Ougolnitsky, 2019).

In our case the set of equilibrium strategies of each influence agent given the principal's strategy is a singleton, therefore, the numerator in (25) is equal to the right hand side of the formula (24).

For the calculation of the denominator in the expression (25) let us assume that each influence agent maximizes not his payoff but the principal's payoff given her strategy, i.e. the influence agent solves the problem

$$
\tilde{J}_{i}\left(\left\{x_{j}(t)\right\}_{j=1}^{n}\right)=\int_{0}^{T} e^{-\rho t} \sum_{j=1}^{n} x_{j}(t) d t \rightarrow \max ,
$$

with conditions (4)-(6). This function is introduced for finding the influence agent stratigies which maximize the payoff function of the Centre.

The Hamilton-Jacobi-Bellman equation has the form

$$
\rho V_{i}-\frac{\partial V_{i}}{\partial t}=\max _{u_{j}^{i}, 1 \leq j \leq n}\left\{\sum_{j=1}^{n} x_{j}(t)+\sum_{j=1}^{n} \frac{\partial V_{j}}{\partial x_{j}}\left[\sum_{k=1}^{m} b_{j}^{k} \sqrt{u_{j}^{k}(t)}+\sum_{l=1}^{n} a_{l j} x_{l}\right]\right\}
$$

with condition $0 \leq \sum_{j=1}^{n} u_{j}^{i}(t) \leq r_{i}(t)$. Maximization by $u_{j}^{i}, j=1,2, \ldots, n, b_{j}^{i} \neq 0$, we receive

$$
u_{j}^{i}(t)=\frac{R_{i}(t)\left(b_{j}^{i} \frac{\partial V_{i}}{\partial x_{j}}\right)^{2}}{\sum_{j=1}^{n}\left(b_{j}^{i} \frac{\partial V_{i}}{\partial x_{j}}\right)^{2}}
$$

and, naturally,

$$
\sum_{j=1}^{n} u_{j}^{i}(t)=R_{i}(t)
$$

Taking the linear Bellman functions

$$
V_{i}(x, t)=\sum_{j=1}^{n} \alpha_{j}^{i}(t) x_{j}+\beta^{i}(t)
$$

we can write the equation (26) with consideration of (27) as

$$
\begin{array}{r}
\rho \sum_{j=1}^{n} \alpha_{j}^{i}(t) x_{j}+\rho \beta^{i}(t)-\sum_{j=1}^{n} \alpha_{j}^{\prime i}(t) x_{j}-\rho \beta^{\prime i}(t)= \\
=\sum_{j=1}^{n} x_{j}(t)+\sum_{l=1}^{n} \sum_{j=1}^{n} \alpha_{j}^{i}(t) a_{l j} x_{l}+\sum_{k=1}^{m} \sum_{j=1}^{n} \alpha_{j}^{i}(t) b_{j}^{k} \frac{\sqrt{\left(R_{k}(t)\right.} \alpha_{j}^{k}(t) b_{j}^{k}}{\sqrt{\sum_{j=1}^{n}\left(\alpha_{j}^{k}(t) b_{j}^{k}\right)^{2}}} .
\end{array}
$$


Equating the coefficients at the first power of $x$ in the left and right hand sides of (28), we receive differential equations for the coefficients $\alpha$ :

$$
\alpha_{j}^{\prime i}(t)-\rho \alpha_{j}^{i}(t)+\sum_{l=1}^{n} \alpha_{l}^{i}(t) a_{j l}=-1,
$$

coinciding with the equations (10) solved by the expression (12).

Choosing the maximal value of the right hand side of (28) in dependence on the sum of $R_{i}(t)$, we have

$$
R_{i}(t)=r_{i}(t),
$$

that is natural because the influence agents care for the principal's payoff instead of their own one. Thus, the strategies of all influence agents are:

$$
u_{j}^{i}(t)=\frac{r_{i}(t)\left(b_{j}^{i} \alpha_{j}(t)\right)^{2}}{\sum_{j=1}^{n}\left(b_{j}^{i} \alpha_{j}(t)\right)^{2}},
$$

and, therefore,

$$
\sum_{j=1}^{n} u_{j}^{i}(t)=r_{i}(t)
$$

where $\alpha_{j}(t), j=1,2, \ldots, n$ are the components of the vector $\alpha(t)$ which is determined by the expression (12).

Let us move to the principal's problem (1)-(2),(5). The condition (5) with consideration of $(30)$ can be immediately written as

$$
\dot{x_{j}}=\sum_{i=1}^{m} \sqrt{r_{i}(t)} b_{j}^{i} \frac{\alpha_{j}(t) b_{j}^{i}}{\sqrt{\sum_{j=1}^{n}\left(\alpha_{j}^{i}(t) b_{j}^{i}\right)^{2}}}+\sum_{l=1}^{n} a_{l j} x_{l} .
$$

The Hamilton-Jacobi-Bellman equation has the form

$$
\begin{array}{r}
\rho V_{0}-\frac{\partial V_{0}}{\partial t}=\max _{r_{i}(t), 1 \leq i \leq m}\left\{\sum_{j=1}^{n} x_{j}(t)-\sum_{i=1}^{m} r_{i}(t)+\right. \\
\left.+\sum_{j=1}^{n} \frac{\partial V_{0}}{\partial x_{j}}\left[\sum_{i=1}^{m} \sqrt{r_{i}(t)} b_{j}^{i} \frac{\alpha_{j}(t) b_{j}^{i}}{\sqrt{\sum_{j=1}^{n}\left(\alpha_{j}^{i}(t) b_{j}^{i}\right)^{2}}}+\sum_{l=1}^{n} a_{l j} x_{l}(t)\right]\right\}
\end{array}
$$

with condition $\sum_{i=1}^{m} r_{i}(t) \leq R, r_{i}(t) \geq 0, i=1,2, \ldots, m$.

Let us use the linear Bellman function:

$$
V_{0}(x, t)=\sum_{j=1}^{n} \alpha_{j}^{0}(t) x_{j}+\beta^{0}(t),
$$

then the equation (31) takes the form

$$
\begin{array}{r}
\rho\left(\beta^{0}+\sum_{j=1}^{n} \alpha_{j}^{0} x_{j}\right)-\left(\beta^{\prime 0}+\sum_{j=1}^{n} \alpha_{j}^{\prime 0} x_{j}\right)=\max _{r_{i}(t), 1 \leq i \leq m}\left\{\sum_{j=1}^{n} x_{j}(t)-\right. \\
\left.-\sum_{i=1}^{m} r_{i}(t)+\sum_{j=1}^{n} \alpha_{j}^{0}\left[\sum_{i=1}^{m} \sqrt{r_{i}(t)} b_{j}^{i} \frac{\alpha_{j}(t) b_{j}^{i}}{\sqrt{\sum_{j=1}^{n}\left(\alpha_{j}(t) b_{j}^{i}\right)^{2}}}+\sum_{l=1}^{n} a_{l j} x_{l}(t)\right]\right\}
\end{array}
$$


with condition $\sum_{i=1}^{m} r_{i}(t) \leq R, r_{i}(t) \geq 0, i=1,2, \ldots, m$.

Let us show that $\alpha_{j}^{0}(t)=\alpha_{j}(t), j=1,2, \ldots, n, t \in T$. Equating in (32) the coefficients at the first power of $x_{j}$ in the left and right hand sides, we receive the differential equation for $\alpha_{j}^{0}(t)$ :

$$
\alpha_{j}^{\prime 0}(t)-\rho \alpha_{j}^{0}(t)+\sum_{l=1}^{n} \alpha_{l}^{0}(t) a_{j l}=-1,
$$

which coincides with (10) for the same boundary conditions

$$
\alpha(T)=0
$$

therefore

$$
\alpha_{j}^{0}(t)=\alpha_{j}^{1}(t)=\ldots=\alpha_{j}^{m}(t)=\alpha_{j}(t), j=1,2, \ldots, n, t \in[0 ; T] .
$$

With consideration of (33) we can rewrite (32) as

$$
\begin{aligned}
\rho\left(\beta^{0}+\sum_{j=1}^{n} \alpha_{j} x_{j}\right)-\left(\beta^{\prime 0}\right. & \left.+\sum_{j=1}^{n} \alpha_{j}^{\prime} x_{j}\right)=\max _{r_{i}(t), 1 \leq i \leq m}\left\{\sum_{j=1}^{n} x_{j}(t)-\sum_{i=1}^{m} r_{i}(t)+\right. \\
& \left.+\sum_{j=1}^{n} \sum_{l=1}^{n} \alpha_{j}(t) a_{l j} x_{l}+\sum_{i=1}^{m} \sqrt{r_{i}(t)} \sqrt{\sum_{j=1}^{n}\left(\alpha_{j}(t) b_{j}^{i}\right)^{2}}\right\}(34)
\end{aligned}
$$

with condition $\sum_{i=1}^{m} r_{i}(t) \leq R, r_{i}(t) \geq 0, i=1,2, \ldots, m$. Notice that the nonconditional maximization of the expression (34) gives the value of resources

$$
r_{i}(t)=\frac{1}{4} \sum_{j=1}^{n}\left(b_{j}^{i} \alpha_{j}(t)\right)^{2} .
$$

Thus, the principal is interested to allocate to each influence agent $i$ the value of resources (35). Therefore if

$$
\sum_{i=1}^{m} \sum_{j=1}^{n}\left(b_{j}^{i} \alpha_{j}(t)\right)^{2} \leq 4 R
$$

then the principal allocates to each influence agent $i$ the value of resources (35). How the principal should allocate the resources if $\sum_{i=1}^{m} \sum_{j=1}^{n}\left(b_{j}^{i} \alpha_{j}(t)\right)^{2}>4 R$ ? Maximizing the right hand side of $(34)$ by $r_{i}(t), i=1,2, \ldots, m$ with condition $\sum_{i=1}^{m} r_{i}(t) \leq R$, we receive

$$
r_{i}(t)=R \frac{\sum_{j=1}^{n}\left(\alpha_{j}(t) b_{j}^{i}\right)^{2}}{\sum_{i=1}^{m} \sum_{j=1}^{n}\left(\alpha_{j}(t) b_{j}^{i}\right)^{2}} .
$$

Thus, in the instants $t \in[0, T]$ when

$$
R \geq \frac{1}{4} \sum_{i=1}^{m} \sum_{j=1}^{n}\left(b_{j}^{i} \alpha_{j}(t)\right)^{2}
$$


the principal's optimal control value is

$$
r_{i}(t)=\frac{1}{4} \sum_{j=1}^{n}\left(b_{j}^{i} \alpha_{j}(t)\right)^{2} .
$$

In the instants $t \in[0, T]$ when

$$
R<\frac{1}{4} \sum_{i=1}^{m} \sum_{j=1}^{n}\left(b_{j}^{i} \alpha_{j}(t)\right)^{2}
$$

the principal's optimal control value is

$$
r_{i}(t)=\frac{\sum_{j=1}^{n}\left(\alpha_{j}(t) b_{j}^{i}\right)^{2}}{\sum_{i=1}^{m} \sum_{j=1}^{n}\left(\alpha_{j}(t) b_{j}^{i}\right)^{2}} R, i=1,2, \ldots, m .
$$

Combining both expressions for $r_{i}(t)$ in the same formula, we receive the final principal's optimal control:

$$
\begin{array}{r}
r_{i}(t)=\frac{\sum_{j=1}^{n}\left(\alpha_{j}(t) b_{j}^{i}\right)^{2}}{\sum_{k=1}^{m} \sum_{j=1}^{n}\left(\alpha_{j}(t) b_{j}^{k}\right)^{2}} \min \left\{\frac{1}{4} \sum_{k=1}^{m} \sum_{j=1}^{n}\left(\alpha_{j}(t) b_{j}^{k}\right)^{2} ; R\right\}, \\
i=1,2, \ldots, m, t \in[0, T],
\end{array}
$$

where

$$
\alpha(t)=\left(e^{(A-\rho I)(T-t)}-I\right)(A-\rho I)^{-1} \epsilon .
$$

Given (36) we can simplify (34) because

$$
\sum_{i=1}^{m} r_{i}(t)=\min \left\{\frac{1}{4} \sum_{i=1}^{m} \sum_{j=1}^{n}\left(b_{j}^{i} \alpha_{j}(t)\right)^{2}, R\right\}
$$

and the last term in (34) is equal to

$$
\sum_{i=1}^{m} \sqrt{\sum_{j=1}^{n}\left(\alpha_{j}(t) b_{j}^{i}\right)^{2}}=\sqrt{\sum_{k=1}^{m} \sum_{j=1}^{n}\left(\alpha_{j}(t) b_{j}^{k}\right)^{2}} \min \left\{\sqrt{\sum_{k=1}^{m} \sum_{j=1}^{n}\left(\alpha_{j}(t) b_{j}^{k}\right)^{2}} ; \sqrt{R}\right\} .
$$

Equating the constant terms in the left and right hand sides of (34), we receive the differential equation for $\beta^{0}(t)$ :

$$
\beta^{\prime 0}(t)-\rho \beta^{0}(t)=\psi(t),
$$

where

$$
\psi(t)=\min \left\{\frac{1}{4} \sum_{k=1}^{m} \sum_{j=1}^{n}\left(b_{j}^{k} \alpha_{j}(t)\right)^{2} ; \sqrt{R \sum_{k=1}^{m} \sum_{j=1}^{n}\left(b_{j}^{k} \alpha_{j}(t)\right)^{2}}-R\right\}
$$


i.e.

$$
\psi(t)=\left\{\begin{array}{c}
\frac{1}{4} \sum_{i=1}^{m} \sum_{j=1}^{n}\left(b_{j}^{i} \alpha_{j}(t)\right)^{2}, \quad \sum_{i=1}^{m} \sum_{j=1}^{n}\left(b_{j}^{i} \alpha_{j}(t)\right)^{2} \leq 4 R, \\
\sqrt{R \sum_{i=1}^{m} \sum_{j=1}^{n}\left(b_{j}^{i} \alpha_{j}(t)\right)^{2}}-R, \sum_{i=1}^{m} \sum_{j=1}^{n}\left(b_{j}^{i} \alpha_{j}(t)\right)^{2}>4 R .
\end{array}\right.
$$

Thus, the equation (37) coincides with the equation (22) for the same boundary conditions, therefore,

$$
\beta^{0}(t)=\int_{t}^{T} e^{-\rho(\tau-t)} \psi(\tau) d \tau
$$

When $t=0$ we have

$$
\beta^{0}(0)=\int_{0}^{T} e^{-\rho \tau} \psi(\tau) d \tau
$$

where $\psi(t)$ is determined by the expression (37).

Thus, the denominator of the expression (25) $\max _{y \in Y} \max _{z \in Z} J_{0}(y, z)$, or the global maximum of the principal's payoff in the case of cooperative behavior, is equal to:

$$
\sum_{j=1}^{n} \alpha_{j}(0) x_{j}(0)+\int_{0}^{T} e^{-\rho \tau} \psi(\tau) d \tau
$$

where $\alpha_{j}(0), j=1,2, \ldots, n$, are determined by the formula (12), and $\psi(\tau)$ is determined by the expression (37).

The system compatibility index in this problem is equal to

$$
S C I=\frac{\sum_{j=1}^{n} \alpha_{j}(0) x_{j}(0)+\int_{0}^{T} e^{-\rho \tau} f(\tau) d \tau}{\sum_{j=1}^{n} \alpha_{j}(0) x_{j}(0)+\int_{0}^{T} e^{-\rho \tau} \psi(\tau) d \tau}<1 .
$$

Notice that the complete system compatibility is achieved only if $f(\tau)=\psi(\tau)$, and this is possible only in the case when for all influence agents the inequality $\sum_{k=1}^{m} \sum_{j=1}^{n}\left(b_{j}^{k} \alpha_{j}(t)\right)^{2} \leq 4 R\left(1+p_{i}\right)^{2}$ is true, and they lack resources for the common purpose.

\section{Conclusion}

We considered a dynamic SPICE-model of resource allocation in a marketing network. The network includes a coordinating principal that allocates resources, several influence agents (competing firms), and basic agents which form the target audience. The model represents a differential Stackelberg game of the principal with the influence agents. In the case of linear functions of private income of the influence agents we found analytically the unique Nash equilibrium in the game of agents and Stackelberg equilibrium in the general game by means of the HamiltonJacobi-Bellman equations. We proved that the opinion value of any basic agent is equal for all influence agents and the principal. Also we proved that the influence agents assign less resources for the marketing efforts than the principal would like.

We plan to spread the received results to the functions of private income in more general form. Besides, it seems rational to consider in more details the firm's utility from buying by the basic agents the goods and services of this specific firm instead of all firms in the totality. 


\section{References}

Agieva, M. T., A.V. Korolev and G. A. Ougolnitsky (2019). Modeling and Simulation of Impact and Control in Social Networks. In: Modelling and Simulation of SocialBehavioural Phenomena in Creative Societies: First International EURO Mini Conference, MSBC 2019. Vilnius, Lithuania, September 18-20, 2019 Proceedings. Communications in Computer and Information Science (Agarwal, N., L. Sakalauskas and G.-W. Weber, eds), Vol. 1079, pp. 29-40.

Anopchenko, T., O. Gorbaneva, E. Lazareva, A. Murzin and G. Ougolnitsky (2019a). Advanced Solow model as a tool for coordination of interests of spatial economic systems' development (on the materials of the South Russian macro-region). Advances in Systems Science and Applications, 19(4), 1-13.

Anopchenko, T., O. Gorbaneva, E. Lazareva, A. Murzin, G. Ougolnitsky and E. Roshchina (2019b). Dynamic Model of Macroregion Subjects' Interests Coordination. In: Proceedings - 2019 1st International Conference on Control Systems, Mathematical Modelling, Automation and Energy Efficiency, SUMMA 2019, art. no. 8947516, pp. 141-146.

Chkhartishvili, A., D. Gubanov and D. Novikov (2019). Social Networks: Models of Information Influence, Control, and Confrontation. Springer Publishers.

Gorbaneva, O. I. (2019). Models of social and private interests combining with independent agents. Automation and Remote Control, 80(9), 1745-1753.

Jackson, M. (2008). Social and Economic Networks. Princeton: Princeton University Press.

Long, N. V. (2010). A Survey of Dynamic Games in Economics. World Scientific Publishing Company.

Nisan, N., T. Roughgarden, E. Tardos and V. Vazirani (2007). Algorithmic Game Theory. Cambridge University Press.

Sedakov A. A. and M. Zhen (2019). Opinion dynamics game in a social network with two influence nodes. Applied Mathematics. Computer Science. Control Processes, 15(1), 118-125.

Sukhinov A. I., G. A. Ougolnitsky and A.B. Usov (2020). Methods of Solving the Theoretic Game Models for Coordinating Interests in Regulating the Fishery Industry. Mathematical Models and Computer Simulations, 12(2), 176-184.

Ugol'nitskii G. A. and A. B. Usov (2020). Dynamic Models for Coordinating Private and Public Interests in Economic Corruption. Journal of Computer and Systems Sciences International, 59(1), 39-48.

Zhen, M. (2019). Stackelberg equilibrium in opinion dynamics game in social network with two influence nodes. In: Contributions to Game Theory and Management. Vol. XII. Collected Papers of the Twelfth International Conference "Game Theory and Management" (Petrosyan, L. and N. Zenkevich, eds), St. Petersburg: Saint Petersburg State University, pp. 29-40. 